\title{
VITAMIN D STATUS IN PATIENTS WITH PSORIASIS
}

\author{
Stoyan Pavlov', Irina Ivanova ${ }^{2}$, Daniela Gerova ${ }^{3}$ \\ ${ }^{1}$ Clinic of Dermatology and Venereology, Medical University of Varna \\ ${ }^{2}$ Clinic of Gastroenterology, Department of Internal Medicine, \\ Medical University of Varna \\ ${ }^{3}$ Clinical Laboratory, Medical University of Varna
}

\begin{abstract}
INTRODUCTION: Vitamin D may act as an immune modulator in psoriasis and be related with the disease characteristics. Our study aimed to evaluate the vitamin D status in patients admitted for diagnostics or exacerbation of psoriasis.

MATERIALS AND METHODS: 92 patients (50 male, 42 female, mean age 55 years) were included in the analysis. Disease severity was assessed by Psoriasis Area and Severity Index (PASI). The type of the disease was classified according to the age at presentation as early (up to 40 years) and late (above 40 years). Serum levels of 25-hydroxyvitamin $\mathrm{D}, 25(\mathrm{OH}) \mathrm{D}$, were tested with an immunofluorescent method (ADVIA Centaur Vitamin D Total, Siemens).

RESULTS: The mean level of 25(OH)D in psoriatic patients was $12.07 \mathrm{ng} / \mathrm{ml}(30.18 \mathrm{nmol} / \mathrm{l})$, found at the low limit of insufficiency; related $95 \%$ CI range was $10.55-13.60 \mathrm{ng} / \mathrm{ml}(26.38-33.99 \mathrm{nmol} / \mathrm{l})$. A total of 45 out of 92 patients (48.9\%) had vitamin D insufficiency (defined as $25(\mathrm{OH}) \mathrm{D}$ level of 10 to $30 \mathrm{ng} / \mathrm{ml}$ ), 44 patients $(47.82 \%)$ had deficiency $(<10 \mathrm{ng} / \mathrm{ml})$ and only in 3 patients $25(\mathrm{OH}) \mathrm{D}$ it was assessed at a level of sufficiency. The Vitamin D level correlates with PASI $(r=-0.508)$, but does not correlate with a C-reactive protein. No significant difference in the vitamin $D$ status was observed in patients with obesity and early type 1 psoriasis.

CONCLUSION: The high prevalence (47.83\%) of vitamin D deficiency in patients with moderate and severe psoriasis infers the role of vitamin $D$ in the pathogenesis of the disease exacerbation. Vitamin $D$ therapeutic supplementation needs to be clarified by further studies.
\end{abstract}

Keywords: psoriasis, vitamin D, vitamin D deficiency

\section{INTRODUCTION}

Psoriasis is a common chronic, autoimmune, inflammatory disease with a global prevalence of $3 \%$. Recently, psoriasis has been associated not only with arthritis but also with disturbances in the lip-

Address for correspondence:

Stoyan Pavlov, MD

Clinic of Dermatology and Venereology

Medical University of Varna,

51 Marin Drinov Str., Varna, Bulgaria

e-mail: stoyanpavlov@abv.bg

Received: February 3, 2016

Accepted: March 7, 2016 id and glucose homeostasis. Patients with psoriasis have an increased risk for cardiovascular incidents and neoplasms. In accordance with these data, psoriasis is accepted as a systemic disease with a typical involvement of the skin and joints (1-3). The concept of the anti-inflammatory and immune-regulating function of vitamin $\mathrm{D}$ was confirmed by multiple current studies. Deficiency and insufficiency of vitamin D were found in many immune-mediated and neoplastic diseases. So, the role of vitamin $\mathrm{D}$ in the pathogenesis of psoriasis, as well as in rheumatoid arthritis, multiple sclerosis, systemic lupus, and inflammatory bowel diseases can be speculated (4-6). The assessment of the blood level of 25-hydroxyvi- 
tamin $\mathrm{D}, 25(\mathrm{OH}) \mathrm{D}$, which is a major circulating metabolite of vitamin $\mathrm{D}$ is an accepted test for a vitamin D status (7). Our study aimed: to investigate the vitamin $\mathrm{D}$ status in patients with Psoriasis vulgaris, consecutively admitted in the Clinic of Dermatology and Venereology - Varna for diagnostics or disease exacerbation; to register the actual severity of psoriasis (PASI) and the type of the disease (early or late, according to age at onset) and to test the relationship between these disease characteristics and the degree of vitamin D insufficiency.

\section{MATERIALS AND METHODS}

92 patients, referred for diagnosis and treatment of psoriasis for a 2-year period from January, 2013 to December, 2014 were included in this retrospective study. Psoriasis vulgaris was established based on clinical manifestation and was verified by skin biopsy and consistent morphology. All patients gave written informed consent for the diagnostic procedures. The type of psoriasis was assessed as an early type 1 (onset of the disease at an age below 40 years) and a late type 2 (when psoriasis was diagnosed at age $>40$ ) (8). Concomitant intake of dietary vitamin D supplements, presence of chronic kidney disease, advanced liver disease and malabsorption excluded patients from the study.

At hospital admission we also assessed disease exacerbation according to Psoriasis Area and Severity Index (PASI). PASI objectively classified the actual severity of psoriasis. The index combines the area of affected skin (head - 10\%; upper extremities - 20\%; lower extremities $40 \%$; trunk - 30\% of whole body surface) and the grade of erythema, desquamation and infiltration or induration (scored for each criteria from 0 to 4). Regarding PASI, the psoriasis was categorized as mild (index $\leq 10)$, moderate $(>10, \leq 30)$ and severe (30 to 72), respectively.

The following data were collected: age at diagnosis and at admission; gender; body mass index (BMI) and waist circumference; C-reactive protein (CRP) and erythrocyte sedimentation rate (ESR), serum level of glucose, lipids, uric acid and creatinine. Overweight and obesity were defined according to WHO definitions as $\mathrm{BMI} \geq 25$ and $\mathrm{BMI} \geq 30$, respectively.

Serum levels of 25(OH)D were calculated by an immunofluorescent test (ADVIA Centaur Vitamin
D Total, Siemens) with assay range of 3.7 to 150ng/ $\mathrm{ml}$ (9.3-375 nmol/l). The vitamin D status was classified as deficiency $(25(\mathrm{OH}) \mathrm{D}$ concentration $<10 \mathrm{ng} /$ $\mathrm{ml} / 25 \mathrm{nmol} / \mathrm{l})$, insufficiency (10-30 ng/ml / 25 -75 $\mathrm{nmol} / \mathrm{l})$, sufficiency $(>30 \mathrm{ng} / \mathrm{ml} / 75 \mathrm{nmol} / \mathrm{l})$ and toxicity (>100ng/ml / 250nmol/l). Serum 25(OH)D levels can be reported in $\mathrm{ng} / \mathrm{ml}$ (common units) or $\mathrm{nmol} / \mathrm{l}$ (SI units) with a conversion formula $1 \mathrm{ng} /$ $\mathrm{ml}=2.5 \mathrm{nmol} / \mathrm{l}(9,10)$.

The continuous variables were expressed as a mean value with a standard deviation. The differences between the variables were analyzed with the Mann-Whitney test and the Kruskal-Wallis test (in analyses of more than 2 independent variables). The relations between $25(\mathrm{OH}) \mathrm{D}$ and disease characteristics were assessed using the Spearman correlation coefficients. The software used for the analysis was GraphPad Prism, a trial 5.0 version.

\section{RESULTS}

The study included 50 male (age 56.69 \pm 13.62 years) and 42 female (age 54.12 \pm 12.11 years) individuals. Prevalence of overweight and obesity was $56 \%$ and 29\%; measured waist circumference was $98.28 \pm 24.45 \mathrm{~cm}$. Psoriatic patients had increased fasting glucose and diabetes $16.3 \%$ and $15.2 \%$, respectively. Total blood cholesterol $>5.2 \mathrm{mmol} / \mathrm{l}$ was observed in $44 \%$ of the study group, but most of them had a borderline elevation and only 9 patients $(10 \%)$ presented with high cholesterol $(>6.2 \mathrm{mmol} / \mathrm{l})$. Similarly, triglycerides above $1.7 \mathrm{mmol} / \mathrm{l}$ and $2.2 \mathrm{mmol} / \mathrm{l}$ were assessed in $25 \%$ and $16.3 \%$ of the patients, respectively. Only 2 patients had both high cholesterol and high triglycerides. $57.6 \%$ of our cases had type 1 psoriasis. The disease was graded as moderate or severe with PASI $32.75 \pm 12.07$ and the mean value of calculated PASI was found at the low limit of the severe forms. Current PASI and CRP level were not significantly different in the type 1 and type 2 psoriatic cases. The patients' characteristics are summarized and shown in Table 1.

The mean level of $25(\mathrm{OH}) \mathrm{D}$ in psoriatic patients was $12.07 \mathrm{ng} / \mathrm{ml}(30.18 \mathrm{nmol} / \mathrm{l})$, found at the low limit of insufficiency; related 95\% CI range was 10.55 $-13.60 \mathrm{ng} / \mathrm{ml}(26.38-33.99 \mathrm{nmol} / \mathrm{l})$. Summary of data on vitamin D status in studied group with moderate and severe psoriasis is presented in Table 2. 
Table 1. Baseline characteristics of patients with psoriasis $(n=92)$

Male/Female

$50 / 42$

Age at investigation, years

BMI

$55.49 \pm 12.96$

$29.92 \pm 7.16$

Waist circumference, $\mathrm{cm}$

$98.28 \pm 24.45$

$\mathrm{ESR}, \mathrm{mm} / \mathrm{h}$

$20.21 \pm 13.23$

CRP, mg/l

$15.10 \pm 28.24$

Serum glucose, $\mathrm{mmol} / \mathrm{l}$

$6.89 \pm 3.83$

Serum cholesterol, $\mathrm{mmol} / \mathrm{l}$

$5.05 \pm 1.06$

$1.60 \pm 0.98$

$319 \pm 98.74$

$53 / 39$

Early/Late type of psoriasis

$32.75 \pm 12.07$

Data have been presented as mean $\pm S D$. Abbreviations used in table 1: SD (standard deviation); BMI (body mass index); ESR (erythrocytes sedimentation rate); $C R P$ (C-reactive protein); $P A S I$ (psoriasis area and severity index)
There is no difference in serum $25(\mathrm{OH}) \mathrm{D}$ in male and female psoriatic patients.

25(OH)D levels were moderately associated with PASI ( $\mathrm{r}=-0.51$, inverse correlation, $\mathrm{p}=0.002)$, but were not significantly correlated to BMI $(\mathrm{r}=-0.09)$, waist circumference $(\mathrm{r}=-0.12)$ and $\mathrm{CRP}(\mathrm{r}=0.27)$.

44 patients had deficiency of $25(\mathrm{OH}) \mathrm{D}$ associated with PASI of $37.73 \pm 10.63 .45$ patients had insufficiency with PASI of $28.82 \pm 11.62$. In only 3 patients $25(\mathrm{OH}) \mathrm{D}$ was assessed in the range of sufficiency and PASI was $18.67 \pm 5.86$. The severity of psoriasis, reflected by PASI, was highest in patients with $25(\mathrm{OH}) \mathrm{D}$ deficiency and the difference of PASI between patients with deficient and insufficient levels of $25(\mathrm{OH}) \mathrm{D}$ was statistically significant $(\mathrm{p}=0.0002)$, Figure 2. However, the difference between the severity of disease in subjects with insufficiency and sufficiency of $25(\mathrm{OH}) \mathrm{D}$ was not statistically significant

Table 2. Vitamin D status of the cohort patients, admitted for evaluation and treatment of moderate to severe Psoriasis vulgaris

\begin{tabular}{lcc|c} 
& Sufficiency & Insufficiency & Deficiency \\
$\mathrm{n}$, (prevalence) & $3(3.26 \%)$ & $45(48.91 \%)$ & $44(47.83 \%)$ \\
Mean 25(OH)D, ng/ml & 36.54 & 15.65 & 6.75 \\
Lower 95\% CI - Upper 95\% CI of mean & $20.51-52.57$ & $14.20-17.10$ & $6.12-7.37$ \\
\hline
\end{tabular}

CI: confidence interval

The assessed levels of 25(OH)D had a seasonal variation: the patients evaluated in the winter and spring had lower levels of 25(OH)D (9.52 and 10.16 $\mathrm{ng} / \mathrm{ml}$ ) than patients admitted in summer and autumn (15.34 and $13.69 \mathrm{ng} / \mathrm{ml}$, respectively), Figure 1. These seasonal variations can be due to the increased vitamin $\mathrm{D}$ synthesis in the skin during summer.

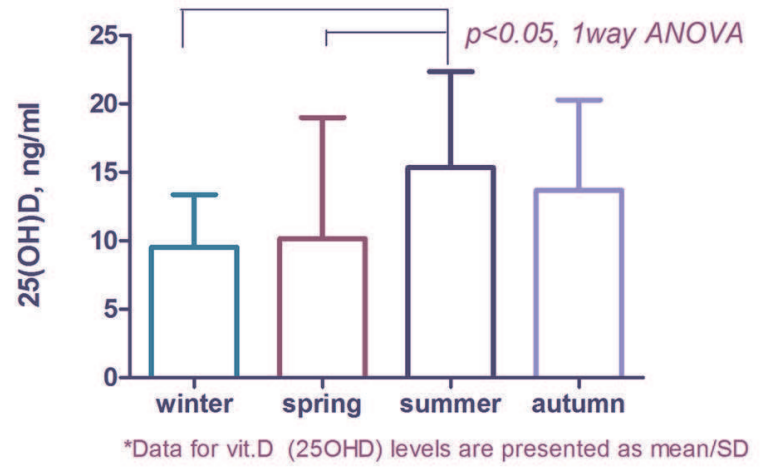

Fig. 1. Level of 25(OH)D in patients with psoriasis, according to the season of investigation

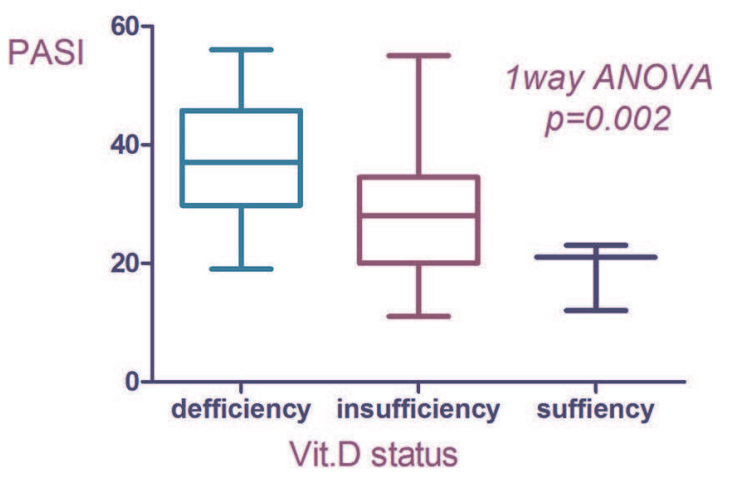

Fig. 2. Severity of psoriasis, assessed by PASI, according to vitamin D status

but we need to take into account the very small group of patients with psoriasis with normal 25(OH)D.

We did not find significant differences in $25(\mathrm{OH}) \mathrm{D}$ levels between patients with early and late type of psoriasis, as well as when obese are compared to normal and overweight patients. 


\section{DISCUSSION}

An increasing number of disorders such as obesity, diabetes, arterial hypertension, rheumatoid arthiritis, with a pathogenic link of chronic inflammation are found to be associated with vitamin $\mathrm{D}$ insufficiency and deficiency. It was accepted that the effect of vitamin $\mathrm{D}$ on immune regulation is due to its interactions with immune cells such as macrophages and lymphocytes. As a result, the production of IL-17, IL-23 and TNF- $\alpha$ is inhibited and the expression of IL- 4 and IL-10 is raised. The immune regulatory role of vitamin $\mathrm{D}$ affects both the innate and the adaptive immune system contributing to the immune tolerance of self-structures. Thus, the deficiency of vitamin D may potentiate immune-mediated inflammation $(11,12)$. Several studies revealed the negative correlation between the grade of rheumatoid arthritis, spondilarthritis and psoriatic arthritis and the serum levels of inflammatory cytokines (IL$1 \alpha$, IL-1 $\beta$, TNF- $\alpha$, TNF- $\beta$, IL-17, IL-23), as well as the serum concentrations of vitamin D (13-15). Vitamin $\mathrm{D}$ deficiency or insufficiency may play a role in the pathogenesis of psoriasis by immune reactions, inducing disturbed differentiation and increased proliferation rate of keratocytes, as well as stimulating angiogenesis $(14,16)$.

Previous clinical analyses have been demonstrating controversies in the relationship between vitamin D deficiency and psoriasis. Population-based study from the United States compared 148 self-reporting cases with mild psoriasis to more than 5800 individuals and failed to establish difference in serum $25(\mathrm{OH}) \mathrm{D}$ and prevalence of vitamin $\mathrm{D}$ deficiency (17). Orgaz-Molina and co-workers, however, observed lower serum levels of vitamin $D$ in patients with psoriasis when compared with controls; negative association between low 25(OH)D levels and both CRP and BMI were found by multiple regression analysis (18). In a study by Gisondi, the participants with psoriasis presented a 2.5 times greater risk of having 25-hydroxyvitamin D deficiency than those without the disease (19). Ricceri et al. found a prevalence of $68 \%$ of vitamin D deficiency and $97 \%$ of insufficiency in the patients with psoriasis studied, while the percentages found in the control group were $10 \%$ of deficiency and approximately $53 \%$ of insufficiency of $25(\mathrm{OH}) \mathrm{D}(20)$.
Accepting the limitation of the absence of control group, our analysis also confirms, that patients with psoriasis are a risk group for vitamin D deficiency and insufficiency. We found $47.83 \%$ prevalence of 25(OH)D deficiency. The observed insufficient rate of $96.74 \%$ is significantly higher, compared to published data from the Bulgarian population on a corresponding prevalence of $75.8 \%$. The vitamin $\mathrm{D}$ status is worse in our patients with moderate to severe psoriasis (mean vitamin D level of $30.18 \mathrm{nmol} / \mathrm{L}$ ) than in the general Bulgarian population (38.75 $\mathrm{nmol} / \mathrm{L})(21)$. Our study demonstrated, that the degree of 25(OH)D deficiency correlates with severity of psoriasis, assessed by PASI.

The effect of vitamin D on immune regulation could suggest the need of supplementation in cases of deficiency and insufficiency. Holick et al, investigating 85 patients with psoriasis established an improvement of PASI, from $18.4 \pm 1.0$ to $9.7 \pm 0.8$, after a 6-month treatment with calcitriol (22). Vitamin D supplementation in deficient patients ( 9 cases with psoriasis and with vitiligo) is efficient in the decrease of PASI and achieved re-pigmentation of the vitiligo areas (4). A trial on the therapeutic role of vitamin D supplementation in our psoriatic patients is needed. The influence of markers related to the immune regulatory function of calcitriol (as interleukins and skin antimicrobial peptides) on the severity of psoriasis should be a matter of interest for further studies.

\section{CONCLUSION}

High prevalence (47.83\%) of vitamin D deficiency in patients with moderate and severe psoriasis infers the role of vitamin $\mathrm{D}$ in the pathogenesis of disease exacerbation. Further studies with larger numbers of patients are required to analyze the pathogenic mechanisms underlying the relationship between 25(OH)D deficiency and psoriasis and the effect of vitamin D supplementation on the clinical course of diseases, based on systemic inflammation.

\section{REFERENCES}

1. Iskandar IYK, Ashcroft DM, Warren RB, et al. Demographics and disease characteristics of patients with psoriasis enrolled in the British Association of Dermatologists Biologic Interventions Register. Br. J. Dermatol. 2015;173:510-518. 
2. Tsoi LC, Spain SL, Knight J, et al. Identification of 15 new psoriasis susceptibility loci highlights the role of innate immunity. Nat. Genet. 2012;44:1341-1348.

3. Malkic Salihbegovic E, Hadzigrahic N, Cickusic AJ. Psoriasis and Metabolic Syndrome. Med. Arch. 2015;69:85-87.

4. Finamor DC, Sinigaglia-Coimbra R, Neves LCM, et al. A pilot study assessing the effect of prolonged administration of high daily doses of vitamin D on the clinical course of vitiligo and psoriasis. Dermatoendocrinol. 2013;5:222-234.

5. Hayes CE, Hubler SL, Moore JR, et al. Vitamin D Actions on CD4(+) T Cells in Autoimmune Disease. Front. Immunol. 2015;6:100.

6. Yap KS, Northcott M, Hoi AB-Y, et al. Association of low vitamin $\mathrm{D}$ with high disease activity in an Australian systemic lupus erythematosus cohort. Lupus Sci. Med. 2015;2:e64.

7. Hollis BW. Circulating 25-hydroxyvitamin D levels indicative of vitamin D sufficiency: implications for establishing a new effective dietary intake recommendation for vitamin D. J. Nutr. 2005;135:317-322.

8. Henseler T, Christophers E. Psoriasis of early and late onset: characterization of two types of psoriasis vulgaris. J. Am. Acad. Dermatol. 1985;13:450-456.

9. Krasowski MD. Pathology consultation on vitamin D testing. Am. J. Clin. Pathol. 2011;136:507-514.

10. Nair R, Maseeh A. Vitamin D: The "sunshine" vitamin. J. Pharmacol. Pharmacother. 2012;3:118-126.

11. Mathieu C, Adorini L. The coming of age of 1,25-dihydroxyvitamin $\mathrm{D}(3)$ analogs as immunomodulatory agents. Trends Mol. Med. 2002;8:174-179.

12. Szodoray $P$, Nakken B, Gaal J, et al. The complex role of vitamin $\mathrm{D}$ in autoimmune diseases. Scand. J. Immunol. 2008;68:261-269.

13. Hong $\mathrm{Q}, \mathrm{Xu}, \mathrm{Xu} \mathrm{S}$, et al. Associations between serum 25-hydroxyvitamin $\mathrm{D}$ and disease activity, inflammatory cytokines and bone loss in patients with rheumatoid arthritis. Rheumatology (Oxford). 2014;53:1994-2001.

14. Balato A, Schiattarella M, Lembo S, et al. Interleukin-1 family members are enhanced in psoriasis and suppressed by vitamin $\mathrm{D}$ and retinoic acid. Arch. Dermatol. Res. 2013;305:255-262.
15. Fu LW, Vender R. Systemic role for vitamin $d$ in the treatment of psoriasis and metabolic syndrome. Dermatol. Res. Pract. 2011;2011:276-279.

16. Creamer D, Sullivan D, Bicknell R, et al. Angiogenesis in psoriasis. Angiogenesis 2002;5:231-236.

17. Wilson PB. Serum 25-hydroxyvitamin D status in individuals with psoriasis in the general population. Endocrine 2013;44:537-539.

18. Orgaz-Molina J, Buendia-Eisman A, ArrabalPolo MA, et al. Deficiency of serum concentration of 25-hydroxyvitamin D in psoriatic patients: a case-control study. J. Am. Acad. Dermatol. 2012;67:931-938.

19. Gisondi P, Rossini M, Cesare A Di, et al. Vitamin D status in patients with chronic plaque psoriasis. $\mathrm{Br}$. J. Dermatol. 2012;166:505-510.

20. Ricceri F, Pescitelli L, Tripo L, et al. Deficiency of serum concentration of 25-hydroxyvitamin D correlates with severity of disease in chronic plaque psoriasis. J. Am. Acad. Dermatol. 2013;68:511-512.

21. Borissova AM, Shinkov A, Vlahov J, et al. Frequency of deficiency, insufficiency and sufficiency of vitamin D in Bulgarian population ( $\geq 20-80$ years old). Endokrinologya 2012;17:122-134.

22. Perez A, Raab R, Chen TC, et al. Safety and efficacy of oral calcitriol (1,25-dihydroxyvitamin D3) for the treatment of psoriasis. Br. J. Dermatol. 1996;134:1070-1078. 